\title{
Current distribution of collective thermal depinning of Josephson vortices in naturally stacked Josephson junctions
}

\author{
Gil-Ho Lee, ${ }^{1}$ Yong-Duk Jin, ${ }^{1}$ and Hu-Jong Lee ${ }^{1,2}$ \\ ${ }^{1}$ Department of Physics, Pohang University of Science and Technology, Pohang 790-784, Republic of Korea \\ ${ }^{2}$ National Center for Nanomaterials Technology, Pohang 790-784, Republic of Korea \\ (Received 10 January 2010; revised manuscript received 9 April 2010; published 7 May 2010)
}

\begin{abstract}
We investigated the thermal-depinning current $\left(I_{c}\right)$ distribution of Josephson vortices (JVs) in naturally stacked $\mathrm{Bi}_{2} \mathrm{Sr}_{2} \mathrm{CaCl}_{2} \mathrm{O}_{8+\delta}$ intrinsic Jospehson junctions in tesla-range magnetic fields and at different field tilt angles from the in-plane position. The $I_{c}$ distribution in the thermal-activation regime contains accurate information on the bias and magnetic-field dependence of the Josephson-vortex pinning potential. In a few-teslarange magnetic field, JVs in a row in a junction, strongly coupled with each other, are pinned or depinned like a single physical entity at a single pinning center. In the best-aligned in-plane magnetic field, the edge pinning is most relevant and is insensitive to the field strength. In the presence of pancake vortices (PVs) in a slightly tilted field, however, the PV pinning deepens the JV pinning potential linearly with the number of PVs.
\end{abstract}

DOI: 10.1103/PhysRevB.81.174508

PACS number(s): 74.25.Wx, 74.50.+r, 74.72. $-\mathrm{h}, 74.25 . \mathrm{F}-$

\section{INTRODUCTION}

The dynamics of Josephson vortices (JVs), magnetic flux quanta formed in a long Josephson junction, is an interesting subject of studies as a JV behaves as a topologically stable quantum particle. JVs have been suggested to be used for the realization of a Josephson vortex qubit. ${ }^{1}$ The macroscopic quantum tunneling and the energy-level quantization for a single JV were experimentally demonstrated in a fieldinduced pinning potential, ${ }^{2}$ which are considered to be an important step toward the qubit realization using JVs. A microsecond range of dephasing time is predicted for JV qubits, which is a few orders of magnitude larger than that of other types of superconducting qubits. ${ }^{3}$ The long dephasing time is owing to the fact that JVs are topologically stable and weakly coupled to the noisy environment. ${ }^{3} \mathrm{JVs}$ were also used to design or realize a microscopic magnetic flux pump, a lens, and a ratchet, ${ }^{4,5}$ where JVs are used to manipulate the dynamics of pancake vortices (PVs) that are present in superconducting electrodes of stacked Josephson junctions in extremely anisotropic layered superconductors, such as $\mathrm{Bi}_{2} \mathrm{Sr}_{2} \mathrm{CaCu}_{2} \mathrm{O}_{8+\delta}(\mathrm{Bi}-2212)$, in the presence of a $c$-axis field component.

For these applications it is essential to control the dynamical state of JVs, which is sensitive to the presence of pinning. In addition to the pinning by PVs, the pinning by the crystal edges is another major source of JV pinning. In this study we investigate the controllability of JV pinning/depinning in densely stacked Josephson junctions formed in Bi-2212 high- $T_{c}$ single crystals with both microresistor-type pinning ${ }^{6}$ by the sample edges (edge pinning) and pinning induced by PVs (PV pinning). PVs in highly anisotropic layered superconductors give adjustable and reversible pinning for manipulating JVs, which is highly demanded for JV-based device applications.

Atomically stacked intrinsic Josephson junctions (IJJs) naturally form in Bi-2212 single crystals. ${ }^{7}$ Compared to the artificially fabricated Josephson junctions, natural IJJs have much higher junction uniformity, which is essential for studying JV dynamics free from effects of junction disorder.
Magnetic vortices are introduced to stacked junctions in the form of JVs in an in-plane magnetic field $\left(H_{\|}\right)$and of PVs in an out-of-plane field component $\left(H_{\perp}\right)$. Thus, both Josephson and pancake vortices coexist in a magnetic field slightly tilted from the in-plane direction. ${ }^{8,9}$

The displacement of PVs in different layers from their equilibrium positions by the in-plane current around a JV increases the elastic energy of the bent flux line between neighboring PVs and results in the mutually attractive pinning force between pancake and Josephson vortices. ${ }^{8}$ Oscillatory zigzag deformation of $\mathrm{PVs}$ induces the frictional damping on flowing JVs, which slows down depinned JVs. ${ }^{10}$ The interaction between pancake and Jospehson vortices is easily controlled by adjusting the number of PVs or the $H_{\perp}$ of an external magnetic field. This provides a means to control the motion of JVs using PVs or vice versa. ${ }^{4,5}$

JVs are also pinned down by the crystal-edge potential barrier. Near the crystal boundary the Josephson current readjusts itself so as to satisfy the boundary condition of vanishing current flow normal to the crystal edge. This boundary condition is satisfied by the presence of an image vortex of opposite polarity outside the crystal, which in turn attracts JVs toward the junction edge and induces the edge pinning. ${ }^{11}$ Local reduction in the Josephson coupling at the junction edge due to the degradation during the sample fabrication, i.e., the appearance of a microresistor, provides another possible cause of the edge pinning. ${ }^{6}$ The control of the JV motion by this microresistor-type pinning potential, once suggested in a Josephson transmission line, ${ }^{12-14}$ can also be adopted to realize JV qubits. . $^{3,15}$

The presence of the edge-pinning potential is confirmed by the oscillatory magnetoresistance of a moving JV lattice (see the lower inset of Fig. 2). As the intervortex spacing of the lattice varies with field the JV distribution periodically matches with the sample edge. This commensurate JV distribution causes slowing down of JVs in the thermally depinned dissipative JV-flow state at sufficiently high temperatures around $50-70 \mathrm{~K}$. Thus the oscillatory behavior of the JV-flow resistance with field has been interpreted in terms of the interaction between JV lattice and the crystal-edge potential. ${ }^{16-19}$ 


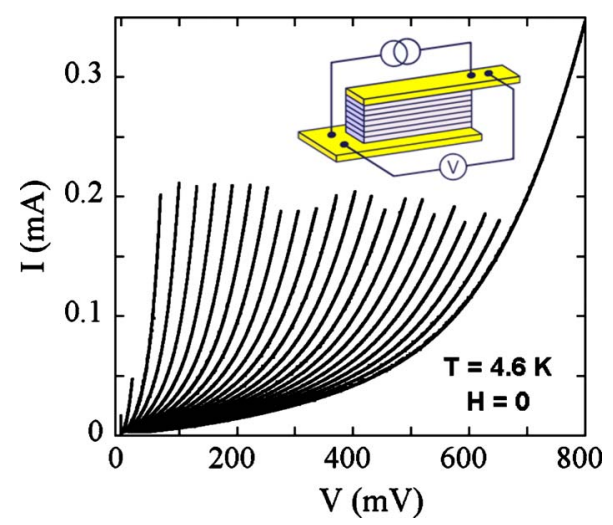

FIG. 1. (Color online) Zero-field current-voltage $(I-V)$ characteristics of the sample stack of $\mathrm{Bi}_{2} \mathrm{Sr}_{2} \mathrm{CaCu}_{2} \mathrm{O}_{8+\delta}$ intrinsic Josephson junctions. Inset: two-probe measurement configuration with the sample stack sandwiched between two gold electrodes.

Depinning of JVs, with increasing the tunneling bias current at sufficiently low temperatures, can be monitored by switching of a JV-flow branch to a higher-voltage branch in the current-voltage $(I-V)$ characteristics. ${ }^{20}$ In this study, we accurately examine the thermally activated and bias-currentdriven depinning process of JVs interacting with PVs and/or the edge pinning potential by monitoring the switching current distribution as a function of the in-plane magnetic-field strength and the field tilt angle from the in-plane position. The JV-flow branches appear in an in-plane magnetic field on an order of a few tesla, where neighboring JVs overlap and are strongly coupled with each other. ${ }^{20,21}$ In consequence, in this high-field range, we find that multiple JVs in a junction (forming a row of JVs) are depinned collectively; depinned like a single JV from a pinning potential. Pinning of JVs by $\mathrm{PVs}$ increases with the $c$-axis magnetic-field strength while pinning of JVs by the edge(s) of a sample (for perfectly aligned in-plane field) is independent of the field strength. This result is consistent with our previous finding of independent depinning of a row of JVs in a junction embedded in naturally stacked IJJs. ${ }^{20}$

\section{EXPERIMENT}

Bi-2212 single crystals were prepared by the conventional self-flux method. ${ }^{22}$ We fabricated a stack of long IJJs with the dimensions of $10 \times 1.5 \times 0.035 \mu^{3}$ sandwiched between two gold electrodes (see the schematic measurement configuration in the inset of Fig. 1) by the double-sidecleaving technique. ${ }^{23,24}$ The sample mounted on a copper block was placed in a ${ }^{4} \mathrm{He}$ cryostat. For the accuracy of thermometry the temperature sensor was embedded in the copper block just beneath the sample. The sample temperature was tuned by the proportional-integral-derivative control of the thermometer with the temperature stability of $0.02 \mathrm{~K}$. All measurement wires were RC filtered with the cut-off frequency of $300 \mathrm{~Hz}$. The sample was field cooled without a bias current when the field strength or the field angle was altered. The in-plane field direction was tuned within the accuracy of $0.01^{\circ}$ by finding the angle for maximum JV-flow

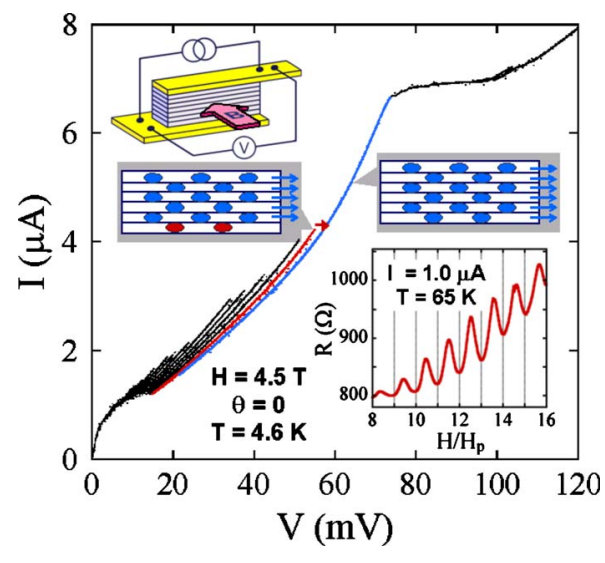

FIG. 2. (Color online) $I-V$ characteristics of Josephson-vortexflow branches in an in-plane magnetic field of $H_{\|}=4.5 \mathrm{~T}$ at $\mathrm{T}$ $=4.6 \mathrm{~K}$. In the schematics, a row of circles with (without) an arrow represents moving (pinned-down) JVs in a junction. Upper inset: schematic of the sample geometry showing the in-plane field direction. Lower inset: oscillatory vortex-flow magnetoresistance in inplane fields for a bias current of $1.0 \mu \mathrm{A}$ at $T=65 \mathrm{~K}$. The field periodicity corresponds to adding one Josephson vortex per two natural Josephson junctions.

resistance at temperature around $65 \mathrm{~K}$ while controlling a stepper motor placed at room temperature. As the pinning on JVs by PVs is minimized at the best-aligned field angle the JV-flow resistance becomes maximum. ${ }^{25}$

\section{RESULTS AND DISCUSSION}

As shown in Fig. 1, the underdamped nature of the IJJs is revealed by hysteretic multiple quasiparticle-tunneling branches in the $I-V$ characteristics of the sandwiched $\mathrm{Bi}$ 2212 stack. The $I-V$ curves were obtained at $4.6 \mathrm{~K}$ by repeated up-down current sweeps. The number of the quasiparticle-tunneling branches ( 23 in our sample) gives the accurate number of IJJs in the stack. The critical current of all the junctions are approximately uniform around $0.2 \mathrm{~mA}$, except for the two lowest-voltage branches. The two branches correspond to the surface junctions that are in contact with the top and bottom gold electrodes with reduced Josephson coupling strength by the proximity effect. ${ }^{22}$

Figure 2 shows the $I-V$ characteristics of the sample stack at $T=4.6 \mathrm{~K}$ in an in-plane magnetic field of $H_{\|}=4.5 \mathrm{~T}$. The field was applied perpendicular to the long side of the sample (see the upper inset of Fig. 2). With the $0.01^{\circ}$ precision of the in-plane field alignment, $H_{\perp}$ is estimated to be $\leq 8 \mathrm{G}$ at the best aligned angle, which gives the negligible pinning effect by PVs. JVs generated in natural JJs by an external in-plane magnetic field form stacked rows of JVs (see the schematics in Fig. 2). In these naturally stacked JJs with minimal intrinsic defects JVs are driven with a high speed by a $c$-axis tunneling bias current $(I)$ that is higher than the depinning or switching current $I_{c}{ }^{21,26}$ However, for $I<I_{c}$, a row of JVs in a junction tend to be pinned down to pinning sources. ${ }^{20}$ The finite vortex-flow voltage of JVs, which are depinned in different rows separately in stacked junctions, generates multiple branches as shown for $V<75 \mathrm{mV}$ in the $I-V$ character- 
istics in Fig. 2. These branches are dubbed as JV-flow branches (JVFBs). We used to interpret the occurrence of JVFBs in terms of the resonance between the JVs and the collective transverse plasma excitation modes in stacked junctions. ${ }^{21,26}$ However, our recent studies on the JV dynamics in Bi-2212 naturally stacked JJs reveal that the JVFBs are generated by separate depinning of a row of JVs in a junction out of the pinning potential. ${ }^{20,27}$

The number of JVFBs is supposed to be same as the number of naturally stacked JJs (23 in our sample). In the highfield range used in this study, however, only 11 JVFBs are visible $^{28}$ in Fig. 2. The $n$th JVFB from right represents the state of $n-1$ pinned rows of JVs and $N-n+1$ flowing rows of JVs among total $N$ junctions in a stack. Thus, for instance, for the second-to-the-rightmost branch, all the rows of JVs are in the flowing state except for one pinned row of JVs. At the bias current $I_{c}$ this remaining row of JVs is depinned, which corresponds to switching to the rightmost branch as denoted by an arrow in Fig. 2. The hysteretic JVFBs indicate the underdamped nature of the dynamics of rows of JVs. Thus, the switching between JVFBs can be reasonably well analyzed in terms of the thermal-activation behavior [see Eq. (4)], where no damping is taken into account. It is also noted that only the JV motion in the underdamped limit can be utilized for the qubit application.

Since the exact shape of the pinning potential is not known, either for PV pinning or for the edge pinning, the simple microresistor-type pinning potential is adopted for the analysis of this study. The microresistor-type pinning is caused by the localized inhomogeneous Josephson coupling in a junction as it perturbs the sine-Gordon equation governing the dynamics of JVs and results in pinning of JVs as described by the potential in Eq. (1). ${ }^{6}$ The intervortex spacing in a row of JVs for the field adopted in this study, $H_{\|}$ $=4.5 \mathrm{~T}$, is about $0.3 \mu \mathrm{m}$, which is shorter than the size of JVs (Ref. 29) $\lambda_{J} \approx 1 \mu \mathrm{m}$. Thus, in this high field $H_{\|}$, dense JVs in a row are strongly coupled with each other and move collectively, which makes it reasonable to model a row JVs as a single physical entity or a particle trapped in a pinning potential

$$
U(q)=U_{0}\left[-\frac{4}{3 \sqrt{3}} \frac{I}{I_{c}} q-\frac{1}{\cosh ^{2}(q)}\right] .
$$

Here, $q$ is the representative coordinate of a JV row (i.e., the coordinate of the JV located at a pinning potential among a row of correlated JVs in a junction) and $U_{0}$ is the pinning barrier in zero bias current. $U_{0}$ and $I_{c}$ are correlated as $U_{0}$ $=\alpha I_{c}$. The parameter $\alpha$, which varies depending on materials and $H_{\|}$value, represents the force exerted to a JV row by a $c$-axis tunneling bias current. Thus, $\alpha$ is proportional to $H_{\|}$as the Lorentz force acted on a JV row linearly increases with increasing $H_{\|}$(or the number of JVs). Figure 3(a) shows the variation in the microresistor-type pinning potential in Eq. (1) for varying current bias. A row of trapped JVs is susceptible to the pinning barrier $\Delta U$, which is approximated for $I \approx I_{c}$.

$$
\Delta U(I)=\frac{8}{9} \sqrt{\frac{2}{3}} U_{0}\left(1-\frac{I}{I_{c}}\right)^{3 / 2}
$$
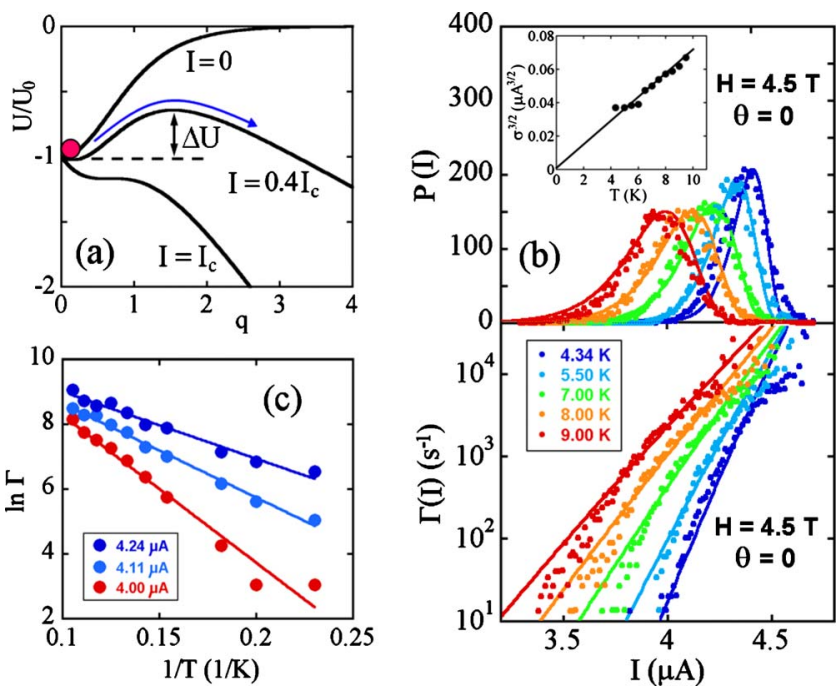

FIG. 3. (Color online) (a) Variation in the microresistor-type pinning potential in Eq. (1) for varying current bias. $\Delta U$ is the pinning potential barrier for a row of Josephson vortices. (b) Depinning current distribution of the second-to-the-rightmost Josephson-vortex-flow branch at different temperatures (upper panel) and the corresponding escaping rate $\Gamma$ (lower panel). Inset: temperature dependence of the standard deviation of the depinning current distribution. Solid lines are best fits to Eqs. (2)-(4). (c) $\ln (\Gamma)$ vs $1 / T$ plot for selected bias currents.

In this study we focus on the $I_{c}$ distribution of the secondto-the-rightmost JVFB to the rightmost JVFB, which is associated with depinning of the single remaining row of pinned JVs. The bias current and the corresponding JV-flow voltage were recorded by using an NI-DAQ board (NI-6281) with the sampling rate of $100 \mathrm{kHz}$ while a sawtoothlike $c$-axis tunneling bias current was applied with a constant ramping rate of $\dot{I}=0.35 \mathrm{~mA} / \mathrm{s}$ and the $50-\mathrm{Hz}$ repetition rate. The current source is battery powered to reduce electrical noise. Each switching of a branch to a neighboring highervoltage branch produces a peak in the differentiated sample voltage during the increasing bias-current sweep. Depinning of the last row of JVs was determined by the highest-voltage (or last differentiated voltage) peak. The value of $I_{c}$ was recorded 10000 times at each temperature with the current resolution $\Delta I$ of $3.5 \mathrm{nA}$.

The upper panel of Fig. 3(b) shows the depinning current distribution $P(I)$ for $H_{\|}=4.5 \mathrm{~T}$ and temperatures ranging from $9.00 \mathrm{~K}$ down to $4.34 \mathrm{~K}$ at $\theta=0$ (i.e., for a perfectly aligned in-plane field). With lowering temperature the current for the most-probable switching gets larger with a narrower distribution. But the asymmetricity of the $I_{c}$ distribution is maintained during the temperature variation. One notices that, at the lowest temperature of $T=4.34 \mathrm{~K}$, the depinning current distribution is broader than the fitting curve. We believe this feature arises as the temperature-independent macroscopic quantum tunneling start emerging. The inset illustrates that the standard deviation of the $I_{c}$ distribution, $\sigma$, well follows the $T^{2 / 3}$ power-law dependence on temperature, which points to the thermally activated collective depinning of JVs from the edge pinning potential. ${ }^{30}$ The convergence of the extrapolation to the origin indicates that the self-heating 

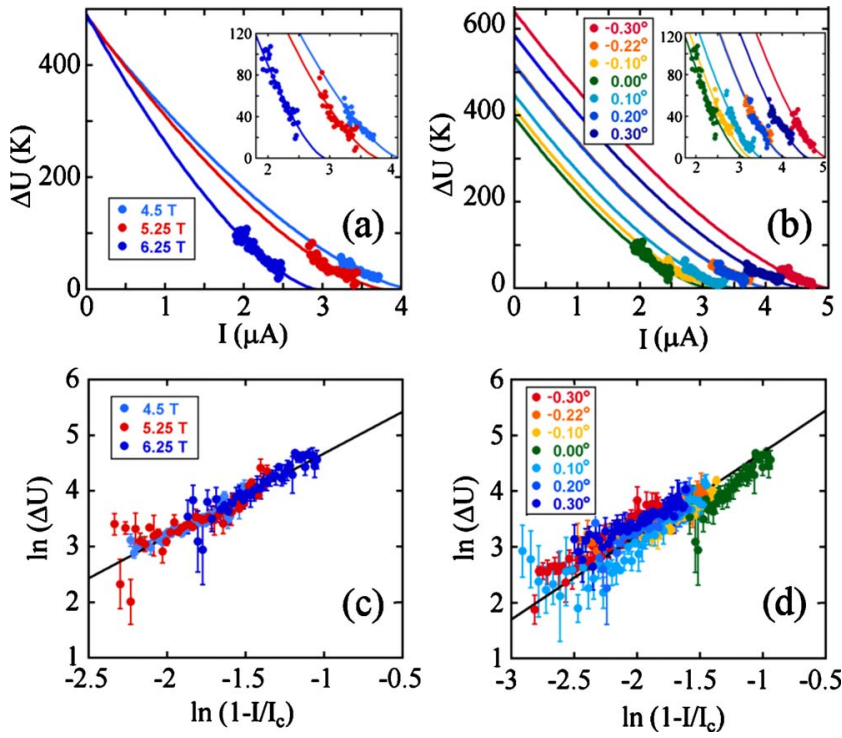

FIG. 4. (Color online) (a) Magnetic-field dependence of the pinning potential barrier $\Delta U$ at the in-plane position. (b) Magneticfield tilt-angle dependence of $\Delta U$ at a fixed magnetic field of $H_{\|}$ $=6.25 \mathrm{~T}$. (c) $\Delta U$ vs $1-I / I_{c}$ in varying fields at the in-plane position. (d) $\Delta U$ vs $1-I / I_{c}$ in $H_{\|}=6.25 \mathrm{~T}$ for varying field tilt angles. Solid line is a guide to eyes for the slope of $3 / 2$.

is negligible. The escaping rate $\Gamma$ as a function of the bias current $I$ is evaluated by converting $P(I)$ by using Eq. (3) below. ${ }^{31}$ Curves $\Gamma(I)$ at various temperatures are shown in the lower panel of Fig. 3(b).

$$
P(I)=\frac{\Gamma(I)}{\dot{I}}\left[1-\int_{0}^{I} P\left(I^{\prime}\right) d I^{\prime}\right] .
$$

At a finite temperature $T$, a row of JVs escapes from the pinning potential of the barrier height $\Delta U$ at the thermal escaping rate $\Gamma$ with an attempt frequency approximated (for $\left.I \approx I_{c}\right)$ by $a_{T}(I)=a_{T 0} \sqrt{8 / 3 \sqrt{3}}\left(1-I / I_{C}\right)^{1 / 4}$ as $^{31}$

$$
\ln \Gamma(I)=\ln a_{T}(I)-\frac{\Delta U(I)}{k_{B}} \frac{1}{T} .
$$

The linearity shown in Fig. 3(c) reconfirms the thermalactivation behavior of $\Gamma$ with the slope corresponding to the potential barrier $\Delta U(I)$ [see Eq. (4)]. In Fig. 3(b) solid lines are best fits to Eqs. (2)-(4) for depinning current distribution $P(I)$ and corresponding escaping rate $\Gamma(I)$ with fitting parameters, $\quad a_{T 0}=80 \mathrm{GHz}, \quad U_{0} / k_{B}=600 \mathrm{~K}, \quad$ and $I_{c}=7.19$ $( \pm 0.70) \mu \mathrm{A}$. It is noted that a set of best-fit parameters satisfy the distribution of $P(I)$ and $\Gamma(I)$ at different temperatures, which implies that the pinning potential adopted by Eqs. (1) and (2) is reasonable.

Figure 4(a) shows $\Delta U$ extracted from the linear fit with three different $H_{\|}$in the best-aligned in-plane field direction, where the edge pinning predominates over the PV pinning. Solid lines are best fits to Eq. (2) with $U_{0}$ and $\alpha$ as best-fit parameters. The values of $U_{0}$ are almost insensitive to $H_{\|}$as $U_{0} / k_{B}=660( \pm 89), 674( \pm 51)$, and $676( \pm 35) \mathrm{K}$ for $H_{\|}$ $=4.5,5.25$, and $6.25 \mathrm{~T}$, respectively, while the corresponding values of $\alpha$ increase linearly with $H_{\|}$as $\alpha=160.3,176.8$, and $229.8 \mathrm{~K} / \mu \mathrm{A}$. The magnetic-field insensitivity of $U_{0}$ indicates that the edge-pinning potential is not affected by the in-plane field strength. This is reasonable because the edge pinning, the predominant pinning source in this case of the best-aligned in-plane fields, depends on the properties of the sample edge but not on the number of JVs. By contrast, $\alpha$ increases with $H_{\|}$as the Lorentz force on a row of JVs increases along with the increase in the number of JVs. Thus, the current-induced decreasing rate of $\Delta U$ gets faster with increasing $H_{\|}$as seen in Fig. 4(a).

Then, fixing $H_{\|}$, the magnetic field angle was slightly tilted up to $0.30^{\circ}$ so as to introduce the PV pinning. For this small tilting $H_{\|}$remains essentially constant while $H_{\perp}$ linearly increases with angle $\theta$ in the rate of $109 \mathrm{G}$ (or $52 \mathrm{PVs}$ in a junction) per $0.1^{\circ}$ for $H_{\|}=6.25 \mathrm{~T}$. Figure 4(b) shows the angle dependence of $\Delta U$ for $H_{\|}=6.25 \mathrm{~T}$, with the best-fit curves. The best fit is obtained with fitting parameter $U_{0}$ and constant $\alpha$, i.e., curves vary almost in parallel with each other. In contrast to the edge pinning, PV pinning increases with tilting angles with a slight asymmetry with respect to the in-plane position.

To ensure the validity of microresistor-type pinning potential, both for edge pinning and PV pinning, we plotted $\Delta U$ vs $1-I / I_{c}$ in a double logarithmic scale in Figs. 4(c) and 4(d). The slopes of variation, with the solid lines as guides to eyes, of both figures [Fig. 4(c) for the edge pinning and Fig. 4(d) for the PV pinning] well agree with the theoretically predicted exponent of $3 / 2$.

In a qualitative sense, a bigger field-tilt angle with more PVs induces stronger pinning of a row of JVs with deepened effective pinning potential. Analysis of PV-induced pinning in a quantitative level, however, is not simple. It has been theoretically proposed that moving JVs cause oscillatory zigzag deformation of PVs. It in turn induces the frictional damping on flowing JVs, which is proportional to the number of PVs. ${ }^{10}$ From this friction one can estimate the number of PVs that actually interact with JVs. Figure 5(a) shows the $I-V$ characteristics taken with slightly tilting the field angles from the perfectly in-plane position for $H_{\|}=4.0 \mathrm{~T}$ and $T$ $=65 \mathrm{~K}$. The high measurement temperature was chosen to focus on the viscous flow motion of thermally depinned JVs while avoiding pinning of JVs. As indicated by an arrow an extra current $[\delta I(V)]$ is required to maintain the JVs velocity, for a particular bias voltage $V$, by the occurrence of the PVinduced frictional damping on JVs.

The parameter $\delta I(V)$ is a direct indicator of the pancake friction, which is related with the voltage $V$ as ${ }^{10}$

$$
\delta I(V)=\Delta G_{\mathrm{JVF}} V=\frac{G_{\mathrm{PV}}}{1+\left(V / V_{r}\right)} V .
$$

Here, $\Delta G_{\mathrm{JVF}}$ is the enhancement of JV-flow conductance due to the pancake friction. $G_{\mathrm{PV}}$ is the quantity related to the viscosity and the spring constant of PVs and the number of 

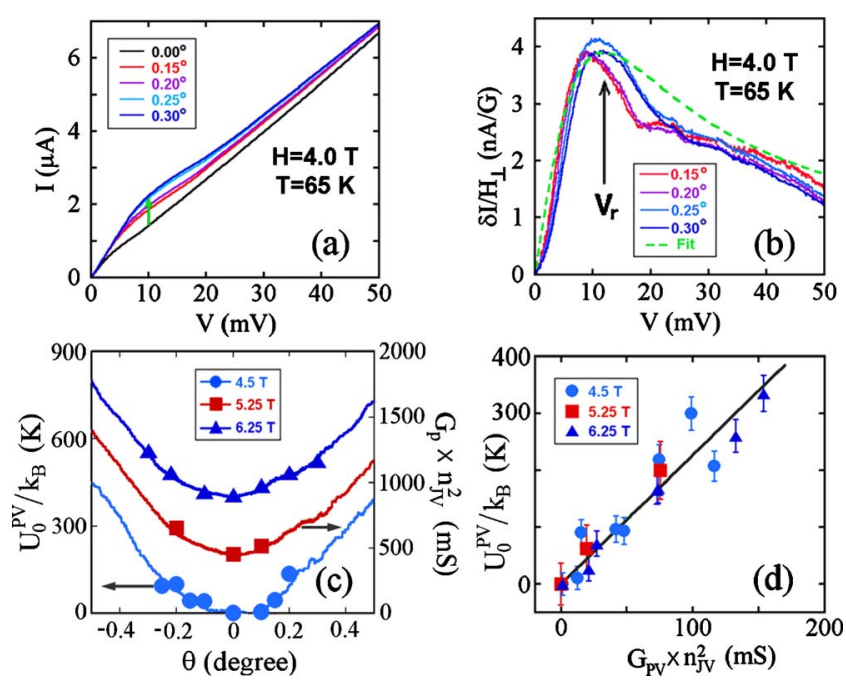

FIG. 5. (Color online) (a) $I-V$ characteristics at $H=4.0 \mathrm{~T}$ and $T=65 \mathrm{~K}$ for varying magnetic-field tilt angles. The arrow indicates an excess current required to compensate the slowing-down of the Josephson vortex motion by pancake vortices. (b) The excess current normalized by the out-of-plane component of magnetic field $H_{\perp}$ with the best-fit to Eq. (5) (dashed curve). (c) Solid lines (symbols) are tilt-angle dependence of $G_{\mathrm{PV}} \times n_{\mathrm{JV}}^{2}$ (of the pinning barrier induced by $\mathrm{PV}$ in the zero-bias limit) in three different magnetic fields. For clarity, each set of data is shifted vertically by $200 \mathrm{~K}$ for $U_{0}^{\mathrm{PV}}$ (or $453 \mathrm{mS}$ for $G_{\mathrm{PV}} \times n_{\mathrm{JV}}^{2}$ ). (d) Relation between enhancement of zero-bias pinning barrier due to $\mathrm{PV} U_{0}^{\mathrm{PV}}(\theta)$ and $G_{\mathrm{PV}} \times n_{\mathrm{PV}}^{2}$. The black solid line is a linear fit with the slope of $2.27( \pm 0.11) \mathrm{K} / \mathrm{mS}$.

vortices involved. $G_{\mathrm{PV}}$ scales with the number of vortices as $G_{\mathrm{PV}} \propto n_{\mathrm{PV}} / n_{\mathrm{JV}}^{2}$, where $n_{\mathrm{PV}}\left(n_{\mathrm{JV}}\right)$ is the number of PVs (JVs). Thus, $G_{\mathrm{PV}} \times n_{\mathrm{JV}}^{2}$ directly represents $n_{\mathrm{PV}}$. As $H_{\perp}$ is roughly proportional to the $n_{\mathrm{PV}}, \delta I(V)$ 's normalized by $H_{\perp}$ for different tilt angles are supposed to merge together as shown in Fig. 5(b). $V_{r}$ is the voltage drop corresponding to the relaxation frequency at which maximum pancake friction is realized [see the arrow in Fig. 5(b)]. The overall behavior is well consistent with theoretical prediction of Eq. (5) [the dotted curve in Fig. 5(b)].

However, $n_{\mathrm{PV}}$ may not be linearly proportional to the $H_{\perp}$ at small tilt angles where screening of magnetic field is not negligible. Note that $H_{\perp}\left(\theta=0.30^{\circ}\right) \approx 210 \mathrm{G}$ is comparable to $H_{c 1}=100-200 \mathrm{G}$ for Bi-2212. ${ }^{32-34} G_{\mathrm{PV}}$ is extracted from the measured $\delta I$ and $V$, along with $V_{r}=12 \mathrm{mV}$ evaluated from theoretical fitting in Fig. 5(b). For a few different values of $H_{\|}$in Fig. $5(\mathrm{c}), G_{\mathrm{PV}} \times n_{\mathrm{JV}}^{2}$ varies parabolically rather than linearly for small field-tilt angles up to $0.30^{\circ}$. Corresponding extra pinning barriers due to PVs, $U_{0}^{\mathrm{PV}}(\theta)=U_{0}(\theta)-U_{0}(\theta=0)$, plotted together in Fig. 5(d). They tend to fall in a single line with a linear relation with $G_{\mathrm{PV}} \times n_{\mathrm{JV}}^{2}$, which confirms that the pinning potential deepens linearly with $n_{\mathrm{PV}}$, although it does not linearly vary with $H_{\perp}$.

\section{SUMMARY}

The depinning current distribution of JVs in naturally stacked IJJs in a long-junction limit, in magnetic fields of a few tesla range and at different field tilt angles from the in-plane position, provides very accurate information on the depinning process of JVs in a junction. A row of strongly coupled JVs in a junction are pinned and depinned collectively. The collective behavior itself is consistent with our previous observation that JVs, either pinned by crystal edge or by the presence of PVs, are collectively depinned in a junction in naturally stacked IJJs but separately from the dynamic state of JVs in other junctions. ${ }^{20}$ More interesting finding of this study is that these strongly coupled JVs show thermally activated depinning characteristics one can expect for a single physical entity from a pinning potential well. The thermally activated depinning of JVs is not sensitive to the detailed shape of the pinning potential. Both the PV pinning and the intrinsic edge pinning of JVs are well described by the microresistor model. For our best-aligned field angle within the accuracy of $0.01^{\circ}$ the pinning is dominated by the intrinsic edge pinning, which is insensitive to the in-plane magnetic field strength. But even for a slight tilt angle of an order of $0.1^{\circ}$ pinning of JVs by PVs becomes significant with the pinning potential deepened as more PVs are introduced to the $\mathrm{CuO}_{2}$ layer of a junction. Quantitative analysis with fitting of the resulting extra current indicates that the depth of the PV pinning potential increases in linear proportion to the number of PVs.

In the study of escaping current distribution of a phase particle from the washboard potential of a Josephson junction or a stack of Jospehson junctions care should be taken to avoid the bias-induced self-heating. Heating reduces the switching current and smears its distribution. It also gives a false characteristic temperature of crossover from the thermal-activation regime to the macroscopic quantum tunneling regime. ${ }^{35,36}$ Thus, to avoid heating, switching current distribution in stacked junctions is usually performed for the switching to the lowest-voltage quasiparticle branch. In the magnetic-field range used this study, however, the JV-flow branches in the low-voltage range are either unidentifiable or unstable to be adopted for studies of the depinning current distribution. In addition, the switching to the highest-voltage branch, adopted in this study, corresponds to the simplest configuration of depinning; depinning of a single row of JVs in a junction. Even in this highest-voltage switching, the selfheating level is sufficiently low as the depinning current of a row of Jospehson vortices in a junction is about two orders of magnitude smaller than the escaping current of phase particle (or the pair-tunneling current in a Jospehson junction).

Although not discussed in the paper, the depinning current distribution of other branches (for instance, between the third and the second to the rightmost branches) leads to qualitatively the same conclusion on the JV depinning characteristics, further confirming the collective thermal depinning in a junction and the independent depinning among different junctions. Macroscopic quantum tunneling of a row of correlated JVs in a junction at lower temperatures and the microwave-induced transition to higher quantized energy levels within the pinning potential well should also be a very interesting subject to be investigated. Accurate information obtained in this study on the depinning characteristics of JVs 
in stacked junctions will be very useful for realizing devices, such as Josephson vortex qubits, based on the interaction between JVs and the pinning elements (PVs and/or microresistors). Moreover, stacked Josephson junctions provide high versatility of controlling the JV-flow dynamics in a reversible way by using the PV pinning.

\section{ACKNOWLEDGMENTS}

This work was supported by National Research Foundation of Korea through Acceleration Research (Grant No. R17-2008-007-01001-0) and the grant under Contract No. 2009-0087091.
${ }^{1}$ A. Wallraff, Yu. Koval, M. Levitchev, M. V. Fistul, and A. V. Ustinov, J. Low Temp. Phys. 118, 543 (2000).

${ }^{2}$ A. Wallraff, A. Lukashenko, J. Lisenfeld, A. Kemp, M. V. Fistul, Y. Koval, and A. V. Ustinov, Nature (London) 425, 155 (2003).

${ }^{3}$ J. H. Kim, R. P. Dhungana, and K.-S. Park, Phys. Rev. B 73, 214506 (2006).

${ }^{4}$ D. Cole, S. Bending, S. Savel'ev, A. Grigorenko, T. Tamegai, and F. Nori, Nature Mater. 5, 305 (2006).

${ }^{5}$ S. Savel'ev and F. Nori, Nature Mater. 1, 179 (2002).

${ }^{6}$ D. W. McLaughlin and A. C. Scott, Phys. Rev. A 18, 1652 (1978).

${ }^{7}$ R. Kleiner, F. Steinmeyer, G. Kunkel, and P. Müller, Phys. Rev. Lett. 68, 2394 (1992).

${ }^{8}$ A. E. Koshelev, Phys. Rev. Lett. 83, 187 (1999).

${ }^{9}$ A. Grigorenko, S. Bending, T. Tamegai, S. Ooi, and M. Henini, Nature (London) 414, 728 (2001).

${ }^{10}$ A. E. Koshelev, Yu. I. Latyshev, and M. Konczykowski, Phys. Rev. B 74, 104509 (2006).

${ }^{11}$ C. P. Bean and J. D. Livingston, Phys. Rev. Lett. 12, 14 (1964).

${ }^{12}$ R. F. Tiwang, P. Woafo, and T. C. Kofané, J. Phys.: Condens. Matter 6, 9745 (1994).

${ }^{13}$ D. R. Gulevich and F. V. Kusmartsev, Phys. Rev. B 74, 214303 (2006).

${ }^{14}$ Y. Zolotaryuk, Eur. Phys. J. B 62, 349 (2008).

${ }^{15}$ T. Kato and M. Imada, J. Phys. Soc. Jpn. 65, 2963 (1996).

${ }^{16}$ S. Ooi, T. Mochiku, and K. Hirata, Phys. Rev. Lett. 89, 247002 (2002).

${ }^{17}$ A. E. Koshelev, Phys. Rev. B 66, 224514 (2002).

${ }^{18}$ M. Machida, Phys. Rev. Lett. 90, 037001 (2003).

${ }^{19}$ Other possible origin of oscillatory behavior of Josephsonvortex-flow resistance in terms of Fiske step was suggested by other authors [A. V. Ustinov and N. F. Pedersen, Phys. Rev. B 72, 052502 (2005)].

${ }^{20}$ Y.-D. Jin, H.-J. Lee, A. E. Koshelev, G.-H. Lee, and M.-H. Bae, EPL 88, 27007 (2009).
${ }^{21}$ M.-H. Bae, J.-H. Choi, and H.-J. Lee, Phys. Rev. B 75, 214502 (2007).

${ }^{22}$ N. Kim, Y.-J. Doh, H.-S. Chang, and H.-J. Lee, Phys. Rev. B 59, 14639 (1999).

${ }^{23}$ H. B. Wang, L. X. You, P. H. Wu, and T. Yamashita, IEEE Trans. Appl. Supercond. 11, 1199 (2001).

${ }^{24}$ M.-H. Bae, H.-J. Lee, J. Kim, and K.-T. Kim, Appl. Phys. Lett. 83, 2187 (2003)

${ }^{25}$ G. Hechtfischer, R. Kleiner, K. Schlenga, W. Walkenhorst, P. Müller, and H. L. Johnson, Phys. Rev. B 55, 14638 (1997).

${ }^{26}$ M.-H. Bae and H.-J. Lee, Int. J. Mod. Phys. B 19, 205 (2005).

${ }^{27}$ In the limit of weak pinning the resonance between Josephson vortices and the collective plasma excitation modes may still be valid.

${ }^{28}$ In a slightly tilted angle the Josephson vortex flow branches are fully identified. Details can be found in Ref. 20.

${ }^{29}$ A. Irie, S. Heim, S. Schromm, M. Mößle, T. Nachtrab, M. Gódo, R. Kleiner, P. Müller, and G. Oya, Phys. Rev. B 62, 6681 (2000).

${ }^{30}$ K. Inomata, S. Sato, K. Nakajima, A. Tanaka, Y. Takano, H. B. Wang, M. Nagao, H. Hatano, and S. Kawabata, Phys. Rev. Lett. 95, 107005 (2005).

${ }^{31}$ T. A. Fulton and L. N. Dunkleberger, Phys. Rev. B 9, 4760 (1974).

${ }^{32}$ K. Tagaya, K. Senda, T. Yosida, N. Fukuoka, and H. Sasakura, Jpn. J. Appl. Phys., Part 2 31, L1170 (1992).

${ }^{33}$ B. vom Hedt and K. Westerholt, Physica C 243, 389 (1995).

${ }^{34}$ M. Nideröst, R. Frassanito, P. Visani, A. C. Mota, and G. Blatter, Physica C 282-287, 1301 (1997).

${ }^{35}$ H. Kashiwaya, T. Matsumoto, H. Shibata, S. Kashiwaya, H. Eisaki, Y. Yoshida, S. Kawabata, and Y. Tanaka, J. Phys. Soc. Jpn. 77, 104708 (2008).

${ }^{36}$ K. Ota, K. Hamada, R. Takemura, M. Ohmaki, T. Machi, K. Tanabe, M. Suzuki, A. Maeda, and H. Kitano, Phys. Rev. B 79, 134505 (2009). 